\title{
ANÁLISE DE PALINOFÁCIES NA AVALIAÇÃO DO POTENCIAL GERADOR DA FORMAÇÃO POJUCA, BACIA DO RECÔNCAVO
}

\author{
HELENA ANTUNES PORTELA, LUZIA ANTONIOLI, RENATA MARINS ALVIM \\ GAMA DE OLIVEIRA, PRISCILA FIGUEIREDO AMARAL \& RODOLFO DINO \\ Departamento de Estratigrafia e Paleontologia, Faculdade de Geologia, UERJ, Rua São Francisco Xavier, 524 Bloco A, \\ Maracanã, Rio de Janeiro, RJ, 20550-900, Brasil.helenaportela@gmail.com,luziaa@uerj.br, \\ ren.gama@gmail.com,priscila.amaral08@gmail.com,dino@uerj.br
}

\begin{abstract}
PALYNOFACIES ANALYSIS IN EVALUATION OF HYDROCARBON POTENTIAL GENERATOR OF THE POJUCA FORMATION, RECÔNCAVO BASIN. This paper deals with the study of source rock potential and its depositional environment. These strata belong to Pojuca Formation and occur in syn-rift sequence of the Early Cretaceous age (Hauterivian-Barremian) in the region of Aramari, a little-explored area of the northwestern Recôncavo Basin. The potential generator of these sediments was evaluated based on studies of palynofacies, Total Organic Carbon (TOC) analysis and RockEval pyrolysis. For the evaluation of the maturation thermal of organic matter of the sediments was used the Spore Coloration Index (SCI); and the palynological study was used to identify the paleoenvironment of these strata. By integration of data from "Rock-Eval" pyrolysis and palynofacies it was possible to identify the prevalence of type II and III kerogen, with varying degrees of quality, allowing for individualization of three palynofacies. The material has predominance of amorphous organic matter of lacustrine origin, with TOC data ranging from $0.95 \%$ to $4.71 \%$. The organic matter is a great potential hydrocarbon generator, indicated by S2 values that reach $26.33 \mathrm{mgHC} / \mathrm{g}$ rock. SCI values between 4.5-5.0 corresponding to 0.5 reflective of vitrinite $(\% \mathrm{Ro})$ demonstrated that the studied section is entering the maturation window. With the data acquired through palynofacies analysis and palynological associations, it can be inferred that a continental deposition environment (deltaiclake) characterizes the sedimentation of the Pojuca Formation with the presence of higrophytic and xerophytic elements, indicating that the vegetation developed in an arid and dry paleoclimate with wet niches locally and latitude next to the present. The data show that the studied area is placed in anoxic conditions containing good quality kerogen and suitable for the generation of hydrocarbons.
\end{abstract}

Key words: palynofacies, Palynology, Pojuca Formation, Early Cretaceous, Recôncavo Basin.

RESUMO - O presente trabalho trata do estudo de rochas potencialmente geradoras de hidrocarbonetos e do seu ambiente deposicional. Estes estratos pertencem à Formação Pojuca e ocorrem na sequência sin-rifte de idade eocretácea (HauterivianoBarremiano) na região de Aramari, uma área pouco explorada do noroeste da Bacia do Recôncavo. O potencial gerador desses sedimentos foi avaliado com base nos estudos de palinofácies, análise de Carbono Orgânico Total (COT) e Pirólise Rock-Eval. Para a avaliação da maturação térmica do material orgânico dos sedimentos fez-se a utilização do Índice de Coloração de Esporos (ICE) e com o estudo palinológico foi possível identificar o paleoambiente destes estratos. Através da integração dos dados de pirólise Rock-Eval e do estudo das palinofácies, foi possível identificar a predominância do querogênio do tipo II e III, com diferentes graus na qualidade, permitindo a individualização de três palinofácies. O material possui predomínio de matéria orgânica amorfa de origem lacustre, com dados de COT variando entre $0,95 \%$ a $4,71 \%$. A matéria orgânica possui um excelente potencial gerador de hidrocarbonetos, indicados por valores de S2 que atingem 26,33 $\mathrm{mgHC} / \mathrm{g}$ rocha. Valores de ICE entre 4,5-5,0, correspondendo a 0,5 de reflectância da vitrinita (\%Ro), demonstraram que a seção estudada se encontra entrando na janela de maturação. Com os dados adquiridos por meio das análises de palinofácies e associações palinológicas, pode-se inferir que a sedimentação da Formação Pojuca é caracterizada por um ambiente de deposição continental (deltaico-lacustre) com a presença de elementos higrofíticos e xerofíticos, indicando uma vegetação que se desenvolveu num paleoclima árido, com nichos úmidos localmente e de latitude próxima ao atual. Os dados revelam que a área estudada se depositou em condições de anoxia, contendo querogênio de boa qualidade e adequada para a geração de hidrocarbonetos.

Palavras-chave: palinofácies, palinologia, Formação Pojuca, Eocretáceo, Bacia do Recôncavo. 


\section{INTRODUÇÃO}

Rochas geradoras de origem lacustre são responsáveis pela procedência de uma parcela significativa das reservas de petróleo em várias regiões do mundo. No Brasil, em particular, estas rochas são responsáveis por cerca de $90 \%$ das reservas de petróleo descobertas até o momento (Mello \& Maxwell, 1990), tendo gerado todo o petróleo das bacias de Campos, Camamu-Almada, Recôncavo, Tucano e parte do petróleo encontrado nas bacias de Santos, Espírito Santo, Cumuruxatiba, Sergipe-Alagoas, Potiguar e Ceará; incluindose aí as acumulações do Pré-sal.

A Bacia do Recôncavo guarda um importante registro geológico, na forma de uma seção lacustre que inclui basicamente folhelhos e arenitos. Seu eficiente sistema petrolífero define a Bacia do Recôncavo como uma das bacias mais prolíficas do Brasil.

A maturação térmica e o tipo de matéria orgânica das rochas geradoras são alguns dos parâmetros que controlam a geração de hidrocarbonetos. Estes parâmetros podem ser investigados através das análises utilizando a palinofácies e dados geoquímicos.

Estudos anteriores, incluindo geoquímicos (Daniel et al., 1989; Figueiredo et al., 1994; Gaglianone \& Trindade, 1988; Mello et al., 1994) e de petrografia orgânica (Balbinot \& Kalkreuth, 2010), identificaram como a principal rocha geradora da Bacia do Recôncavo a Formação Candeias, mas acredita-se que nesta bacia possa haver outros horizontes sedimentares com um potencial gerador.

Para alcançar o objetivo de inferir o potencial gerador de hidrocarbonetos na Formação Pojuca, o presente trabalho teve como objetivo principal o estudo das palinofácies associadas às técnicas de geoquímica orgânica, assim como estudos palinológicos na caracterização do ambiente de deposição dos sedimentos. Estes estudos foram realizados em rochas cretáceas na região de Aramari (Formação Pojuca) com amostras coletadas do poço 9-FBA-79-BA da Bacia do Recôncavo, Nordeste do Brasil (Figura 1).

\section{CONTEXTO REGIONAL}

A Bacia do Recôncavo está localizada no Nordeste do Brasil, e faz parte do sistema rifte Recôncavo-Tucano-Jatobá (Figura 1). A bacia ocupa uma área de aproximadamente $11.500 \mathrm{~km}^{2}$, onde a primeira descoberta de petróleo no Brasil foi há cerca de 70 anos e até hoje continua a ser um produtor de hidrocarbonetos (Silva et al., 2007).

Segundo Destro et al. (2003), esta bacia é um aulacógeno, caracterizado por estruturas de graben assimétricos formados durante o desmembramento do Gondwana durante o Neocomiano/Eocretáceo. Os limites se dão ao sul por uma zona de transferência E-W (Sistema de Falhas da Barra), que a separa da Bacia de Camamu; a leste, por afloramentos pré-cambrianos, através dos sistemas de falhas de Salvador,

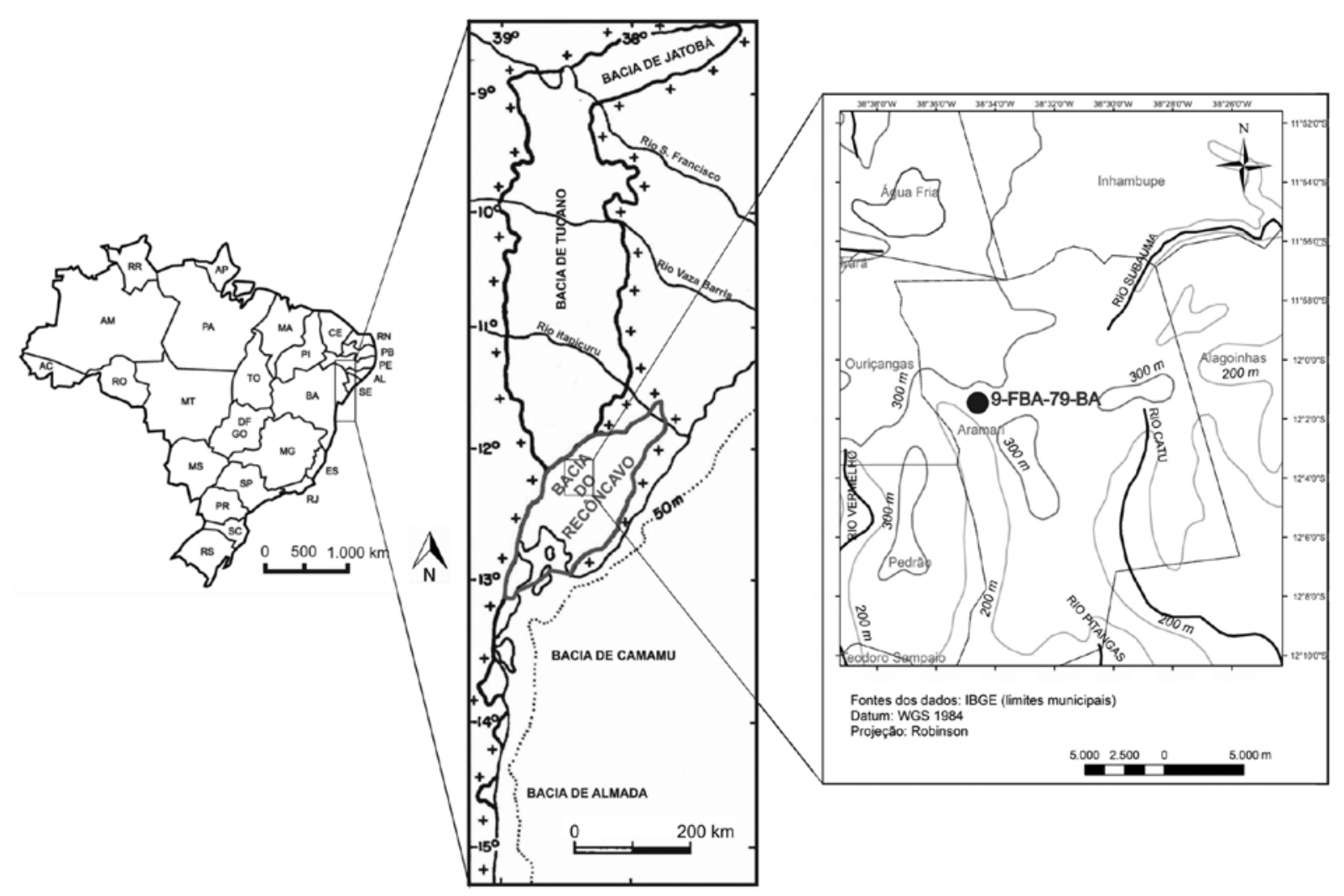

Figura 1. Mapa de localização, limites e arcabouço estrutural da Bacia do Recôncavo. Modificado de Figueiredo et al. (1994).

Figure 1. Location map, limits and structural framework of the Reconcavo Basin. Modified from Figueiredo et al. (1994). 
sendo este o mais expressivo, podendo atingir até $6.000 \mathrm{~m}$ de rejeito; a oeste, pela Falha de Maragogipe, associada à borda flexural do meio-graben; e ao norte pelo Alto de Aporá, que a separa da Bacia do Tucano (Silva et al., 2007).

A sedimentação ocorreu durante os estágios sin-rifte e pós-rifte, de desenvolvimento da bacia, resultando em uma bacia com sedimentação máxima de até $6.500 \mathrm{~m}$ no Baixo Camaçari, no compartimento do Sul (Silva et al., 2007). A fase de pré-rifte está relacionada com os estágios iniciais da crosta flexural e inclui três ciclos flúvio-deltaicos, separados por transgressões lacustres de caráter regional. A sedimentação nesta fase ocorreu durante as condições de clima árido, com a deposição de arenitos e folhelhos fluviais e lacustres.

O desenvolvimento da sequência rifte durante o Eocretáceo ocorreu em um período mais úmido, com forte tectonismo, representado por um sistema fluvial-deltaicolacustre. Durante esta fase foram depositados folhelhos lacustres, arenitos turbidíticos e deltaicos, siltitos, carbonatos e conglomerados perto das margens da bacia, intercalados com sedimentos mais finos (Caixeta et al., 1994; Figueiredo et al., 1994; Silva et al., 2007). A fase pós-rifte começou no Aptiano e corresponde a sedimentação de conglomerados e arenitos aluviais (Magnavita et al., 2005).

Durante a fase de ambiente lacustre anóxico foram depositados sedimentos da Formação Candeias, tendo o Membro Gomo (Rio da Serra) como principal fonte de hidrocarbonetos da Bacia do Recôncavo (Daniel et al., 1989; Figueiredo et al., 1994; Mello et al., 1994), com rochas predominantemente pelíticas, compostas por folhelhos negros intercalados com camadas finas de carbonatos e arenito fino.

Segundo Magnavita (1992) e Silva et al. (2007), o preenchimento sedimentar da Bacia do Recôncavo pode ser dividido em quatro estágios distintos: sinéclise, prérifte, rifte e pós-rifte. No presente trabalho os termos são aplicados e o estrato do presente estudo (Formação Pojuca) está inserido na supersequência rifte. $\mathrm{O}$ início do rifte ocorreu há aproximadamente $144 \mathrm{Ma}$, durante o Berriasiano, já a deposição sedimentar da fase rifte durou $22 \mathrm{Ma}$, desde o início do Barriasiano ao final do Barremiano. O arcabouço estrutural da Bacia do Recôncavo foi determinado durante esse período (Magnavita, 1992).

Durante uma fase inicial de bacia faminta, foram depositados folhelhos lacustres e turbiditos do Membro Gomo (Formação Candeias). Esta deposição ocorreu inicialmente em lago restrito, o qual foi posteriormente ampliado durante a deposição da Formação Maracangalha (Membros Pitanga e Caruaçu) devido à intensa atividade tectônica (Magnavita, 1992). Uma atenuação da atividade tectônica com consequente diminuição na taxa de subsidência (indicado pela presença de carbonatos oncolíticos no topo da Formação Maracangalha) permitiu que deltas progradassem através das plataformas existentes ao longo das margens flexurais, preenchendo os depocentros com arenitos deltaicos das formações Marfim e Pojuca. O peso exercido por esses pacotes de arenito aliado a fisiografia da bacia como um meio-graben, promoveram a pressurização dos folhelhos da Formação Maracangalha resultando em diápiros de folhelhos associados a falhas de crescimento (Magnavita, 1992).

A Formação Pojuca é constituída por arenitos muito finos a médios, às vezes sílticos, calcíferos e folhelhos cinza, siltitos e biocalcarenitos ostracoidais, que também são de origem flúvio-deltaica (Caixeta et al., 1994).

\section{MATERIAL E MÉTODOS}

Para os estudos de palinofácies e geoguímica orgânica foram utilizadas 34 amostras e para o estudo palinológico 17 amostras da Formação Pojuca. As amostras são de sondagem rasa de testemunhagem contínua pertencente ao poço 9-FBA79-BA (99,0 m) (Figura 2), perfurado pela antiga Petromisa no ano de 1982, visando avaliar o potencial exploratório de sulfetos, localizado na porção NE do Estado da Bahia, na cidade de Aramari (Figura 1).

Inicialmente foi realizada uma coleta sistemática das amostras, com cuidado da amostragem recair preferencialmente nos sedimentos de granulometria fina e de coloração mais escura, por constituírem sedimentos ricos em matéria orgânica. As amostras foram registradas entre os números 2012031 a 2012065 no Laboratório de Palinomacerais (LBPM) da Faculdade de Geologia da Universidade do Estado do Rio de Janeiro, onde as mesmas sofreram processos de tratamentos físicos e químicos, além da preparação das lâminas de palinofácies e palinológicas. Já as análises de Carbono Orgânico Total (COT) e de pirólise Rock-Eval foram realizadas no Laboratório de Geoquímica (LGQM), localizado na mesma universidade.

A preparação do material utilizou os procedimentos palinológicos não oxidativos, padrões descritos por Tyson (1995) e Mendonça Filho et al. (2002). Essas amostras foram tratadas com ácido clorídrico e fluorídrico para eliminação da fração mineral, e a separação da matéria orgânica do resíduo mineral e a sua concentração foi realizada com a utilização de cloreto de zinco. Com o material orgânico isolado, foram confeccionadas as lâminas e posteriormente analisadas (seguindo o método de Tyson, 1995) em luz branca transmitida e luz ultravioleta incidente (fluorescência).

No estudo quantitativo os dados percentuais foram obtidos pela contagem de no mínimo 300 partículas do material orgânico, com dimensões superiores a $10 \mu \mathrm{m}$ (Tyson, 1995). Os grupos do material orgânico são contados e separados em três grupos principais: fitoclastos, palinomorfos e matéria orgânica amorfa, proposto por Tyson (1995). Além das proporções absolutas e relativas, o estado de preservação também foi levado em consideração para as análises das palinofácies. Para o índice de alteração térmica através do Índice de coloração de Esporos, foi utilizada a técnica desenvolvida por Barnard et al. (1981), a qual apresenta uma escala de 1 a 10 , em intervalos de 0,5 . No tratamento dos dados palinológicos fez-se necessário a utilização da técnica sugerida por Traverse (1988).

Os resultados de COT expressam a quantidade de matéria orgânica presente nos sedimentos e as análises foram obtidas com o forno de indução do determinador de carbono da 


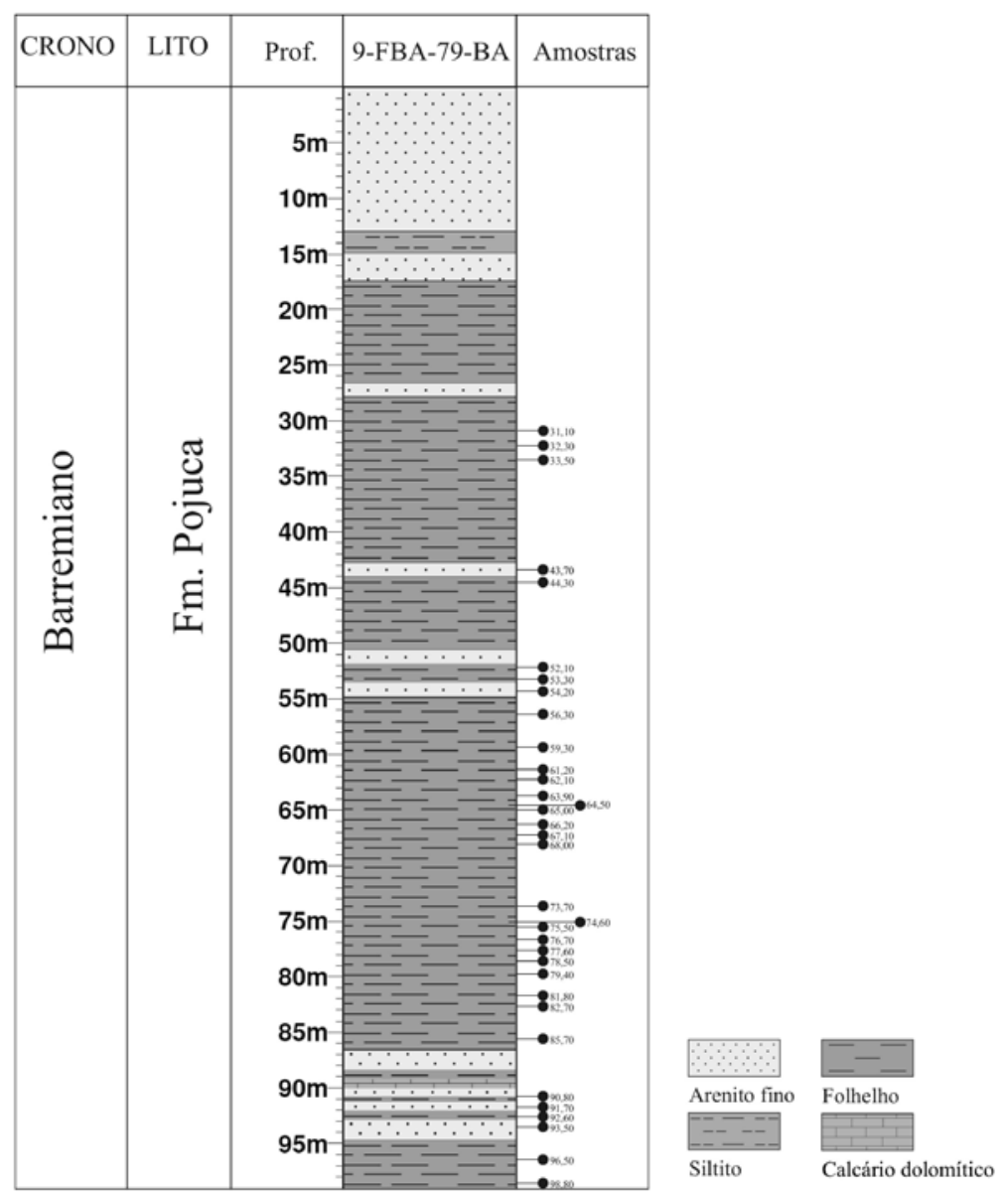

Figura 2. Distribuição das amostras no poço 9-FBA-79-BA mostrando suas principais características litológicas e sedimentológicas.

Figure 2. Distribution of samples in the 9-FBA-79-BA well showing its main lithological and sedimentological features.

LECO, onde foram adotados os métodos ASTM D 4239 (American Society for Testing and Materials - ASTM, 2008) e NCEA-C-1282 (United States Environmental Protection Agency, Schumacher, 2002). Para as análises de pirólise RockEval, que fornece informações sobre a quantidade e o tipo de matéria orgânica em uma rocha sedimentar, além do nível de maturação orgânica (Espitalié et al., 1977), as amostras de cada intervalo foram pulverizadas com cerca de $100 \mathrm{mg}$, utilizando o aparelho ROCK-EVAL 6 CLASSIC, seguindo técnicas descritas por Behar et al. (2001).

Após a contagem dos componentes orgânicos particulados, os valores absolutos foram recalculados para valores percentuais e normalizados a $100 \%$. Para uma melhor resolução das informações obtidas, foram gerados gráficos para denotar as variações nas assembleias de palinofácies e dos dados geoquímicos ao longo do intervalo estudado.

\section{RESULTADOS}

\section{Palinologia}

Foi realizado recentemente por Portela (2012) um trabalho palinológico detalhado sobre os sedimentos da Formação Pojuca na Bacia do Recôncavo, onde o pacote rochoso estudado e a associação palinoflorística mostraram ser constituídos, em sua maior parte, por espécies típicas do Cretáceo Inferior, porém com ampla distribuição vertical, sendo poucas as espécies com alto valor cronoestratigráfico. Deste modo, sobre aspectos qualitativos e quantitativos, o presente trabalho apresentou a mesma composição palinoflorística contida nos depósitos cronocorrelatos presentes nas bacias costeiras brasileiras. Nesses depósitos analisados em Portela (2012), constatou-se a presença das espécies Dicheiropollis etruscus e Vitreisporites pallidus, permitindo o posicionamento na biozona Vitreisporites pallidus (Beurlen et al., 1992).

No presente estudo os dados palinológicos foram utilizados para determinar o ambiente dos palinomorfos recuperados, onde os mesmos se encontram bem conservados (Figura 3). Com este resultado foi possível observar variações das espécies encontradas nas seções estudadas e através das análises quantitativas e de identificação/classificação dos palinomorfos em microscopia óptica de luz branca transmitida, foi possível inferir a palinoflora da época e estabelecer o tipo de ambiente deposicional.

Foram identificadas 25 espécies de palinomorfos correspondentes a 17 gêneros em toda a associação (Figura 4). A seção sedimentar analisada apresenta uma palinoflora rica e diversificada, com elementos típicos de uma palinoflora 


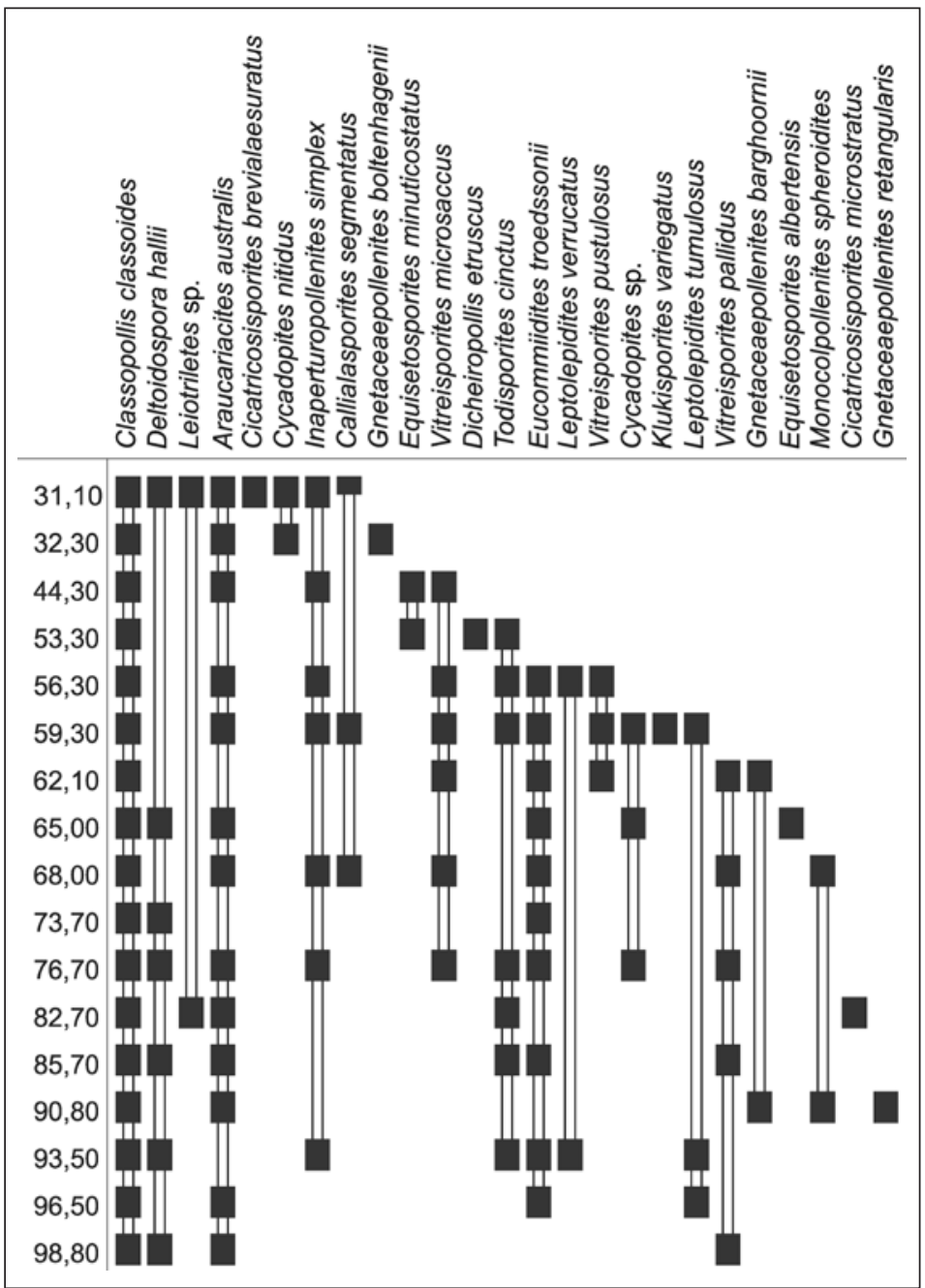

Figura 3. Distribuição vertical dos palinomorfos recuperados do poço 9-FBA-79-BA.

Figure 3. Vertical distribution of palynomorphs recovered from the 9-FBA-79-BA well.

gondwânica. Portanto, a composição da palinoflora do poço 9-FBA-79-BA pode ser subdividida nos seguintes grupos taxonômicos: (i) briófitas (musgos e hepáticas); (ii) pteridófitas, os quais são compostos principalmente por esporos de samambaias e avencas, incluindo também os esporos de licófitas e esfenófitas; (iii) gimnospermas, que incluem grãos de pólen monossacados, bissacados, inaperturados, rimulados, poliplicados e monosulcados.

Em termos de representatividade dentro da seção estudada, de um modo geral, predomina o grupo das gimnospermas seguido pelos esporos triletes (grupo das briófitas e pteridófitas). Em relação às identificações das prováveis afinidades botânicas dos palinomorfos mais representativos da associação recuperada, é possível concluir que a palinoflora identificada foi produzida por uma paleoflora complexa, dominada por gimnospermas e pteridófitas, onde se destaca a grande presença de grãos de pólen Rimulados, representados pela espécie Classopollis classoides.

Portanto, a palinoflora que caracteriza a seção estudada é muito similar às associações contemporâneas, essencialmente gimnospérmicas, pertencentes à mesma faixa microflorística de outras regiões. Neste sentido, insere-se perfeitamente na Província Microflorística Dicheiropollis/Afropollis (Herngreen et al., 1996).

\section{Carbono orgânico total e pirólise Rock-Eval}

$\mathrm{O}$ material analisado apresentou teores significativos de COT variando de $0,95 \%$ a $4,71 \%$, que reflete uma boa quantidade de matéria orgânica depositada. Os resultados da pirólise Rock-Eval (Tabela 1) revelam que os valores de $\mathrm{S} 1$ (quantidade de hidrocarbonetos presentes nas amostras) são baixos e variam de 0,03 a $1,73 \mathrm{mg} \mathrm{HC} / \mathrm{g}$ Rocha e os valores de $\mathrm{S} 2$ (quantidade de hidrocarboneto que as amostras produziram durante a pirólise) alcançam valores de até 26,33 $\mathrm{mg} \mathrm{HC} / \mathrm{g}$. Em relação ao índice de hidrogênio (IH) os valores variam de 150,34 a 559,02 $\mathrm{mg} \mathrm{HC/g}$ de COT e nos de índice de oxigênio (IO) são baixos e variam de 40,78 a 91,06 mg de $\mathrm{CO} 2 / \mathrm{g}$ de COT.

Para classificar o tipo de querogênio deste trabalho foi confeccionado o diagrama binário IHxIO, tipo Van Krevelen (Espitalié et al., 1977), o qual foi classificado como sendo o querogênio do tipo II e III (Figura 5). 
Tabela 1. Resultados de COT (\%) e pirólise Rock-Eval das amostras analisadas do poço 9-FBA-79-BA.

Table 1. Results of the TOC (\%) and "Rock-Eval" pyrolysis of the samples of the 9-FBA-79-BA well.

\begin{tabular}{|c|c|c|c|c|c|c|}
\hline $\begin{array}{c}\text { Amostra } \\
\text { Profundidade (m) }\end{array}$ & $\begin{array}{c}\mathrm{COT} \\
\%\end{array}$ & $\begin{array}{c}\mathrm{S} 1 \\
\mathrm{MgHC} / \mathrm{gR}\end{array}$ & $\begin{array}{c}\mathrm{S} 2 \\
\mathrm{MgHC} / \mathrm{gR}\end{array}$ & $\begin{array}{c}\mathrm{S} 3 \\
\mathrm{MgCO}_{2} / g R\end{array}$ & $\begin{array}{c}\mathrm{IH} \\
\mathrm{MgHC} / \mathrm{COT}\end{array}$ & $\begin{array}{c}\mathrm{IO} \\
\mathrm{MgCO}_{2} / \mathrm{gR}\end{array}$ \\
\hline 31,10 & 2,06 & 0,07 & 3,89 & 0,84 & 188,83 & 40,78 \\
\hline 32,30 & 0,95 & 0,03 & 1,31 & 0,52 & 137,89 & 54,74 \\
\hline 33,50 & 1,31 & 0,06 & 3,21 & 0,72 & 245,04 & 54,96 \\
\hline 43,70 & 3,31 & 0,21 & 10,02 & 1,36 & 302,72 & 41,09 \\
\hline 44,30 & 1,49 & 0,10 & 2,24 & 0,96 & 150,34 & 64,43 \\
\hline 52,10 & 2,02 & 0,22 & 4,00 & 1,39 & 198,02 & 68,81 \\
\hline 53,30 & 1,91 & 0,11 & 3,59 & 1,20 & 187,96 & 62,83 \\
\hline 54,20 & 2,31 & 0,15 & 4,91 & 1,76 & 212,55 & 76,19 \\
\hline 56,30 & 2,26 & 0,12 & 3,69 & 1,83 & 163,27 & 80,97 \\
\hline 59,30 & 1,87 & 0,08 & 3,50 & 1,29 & 187,17 & 68,98 \\
\hline 61,20 & 3,74 & 0,35 & 11,95 & 2,01 & 319,52 & 53,74 \\
\hline 62,10 & 3,52 & 0,21 & 9,99 & 2,07 & 283,81 & 58,81 \\
\hline 63,90 & 4,21 & 0,31 & 13,23 & 2,17 & 314,25 & 51,54 \\
\hline 64,50 & 3,80 & 0,19 & 12,42 & 1,83 & 326,84 & 48,16 \\
\hline 65,00 & 3,96 & 0,55 & 14,00 & 1,73 & 353,54 & 43,69 \\
\hline 66,20 & 4,47 & 0,68 & 15,14 & 2,77 & 338,70 & 61,97 \\
\hline 67,10 & 4,29 & 1,73 & 15,00 & 2,84 & 349,65 & 66,20 \\
\hline 68,00 & 3,41 & 0,34 & 10,47 & 1,64 & 307,04 & 48,09 \\
\hline 73,70 & 4,38 & 0,62 & 23,00 & 2,16 & 525,11 & 49,32 \\
\hline 74,60 & 4,49 & 0,61 & 23,14 & 2,56 & 515,37 & 57,02 \\
\hline 75,50 & 2,24 & 1,08 & 6,54 & 1,56 & 291,96 & 69,64 \\
\hline 76,70 & 3,89 & 0,40 & 17,88 & 2,34 & 459,64 & 60,15 \\
\hline 77,60 & 4,71 & 0,78 & 26,33 & 2,30 & 559,02 & 48,83 \\
\hline 78,50 & 3,00 & 0,54 & 11,42 & 1,61 & 380,67 & 53,67 \\
\hline 79,40 & 4,10 & 0,55 & 20,86 & 1,98 & 508,78 & 48,29 \\
\hline 81,80 & 3,86 & 0,67 & 17,45 & 1,89 & 452,07 & 48,96 \\
\hline 82,70 & 1,23 & 0,18 & 1,98 & 1,12 & 160,98 & 91,06 \\
\hline 85,70 & 3,34 & 0,47 & 13,24 & 1,81 & 396,41 & 54,19 \\
\hline 90,80 & 3,62 & 0,46 & 17,94 & 1,70 & 495,58 & 46,96 \\
\hline 91,70 & 2,16 & 1,06 & 8,30 & 1,02 & 384,26 & 47,22 \\
\hline 92,60 & 2,37 & 0,37 & 9,71 & 1,01 & 409,70 & 42,62 \\
\hline 93,50 & 2,88 & 0,20 & 12,43 & 1,51 & 431,60 & 52,43 \\
\hline 96,50 & 1,70 & 0,11 & 4,35 & 0,82 & 255,88 & 48,24 \\
\hline 98,80 & 1,78 & 0,84 & 4,59 & 1,19 & 257,87 & 66,85 \\
\hline
\end{tabular}




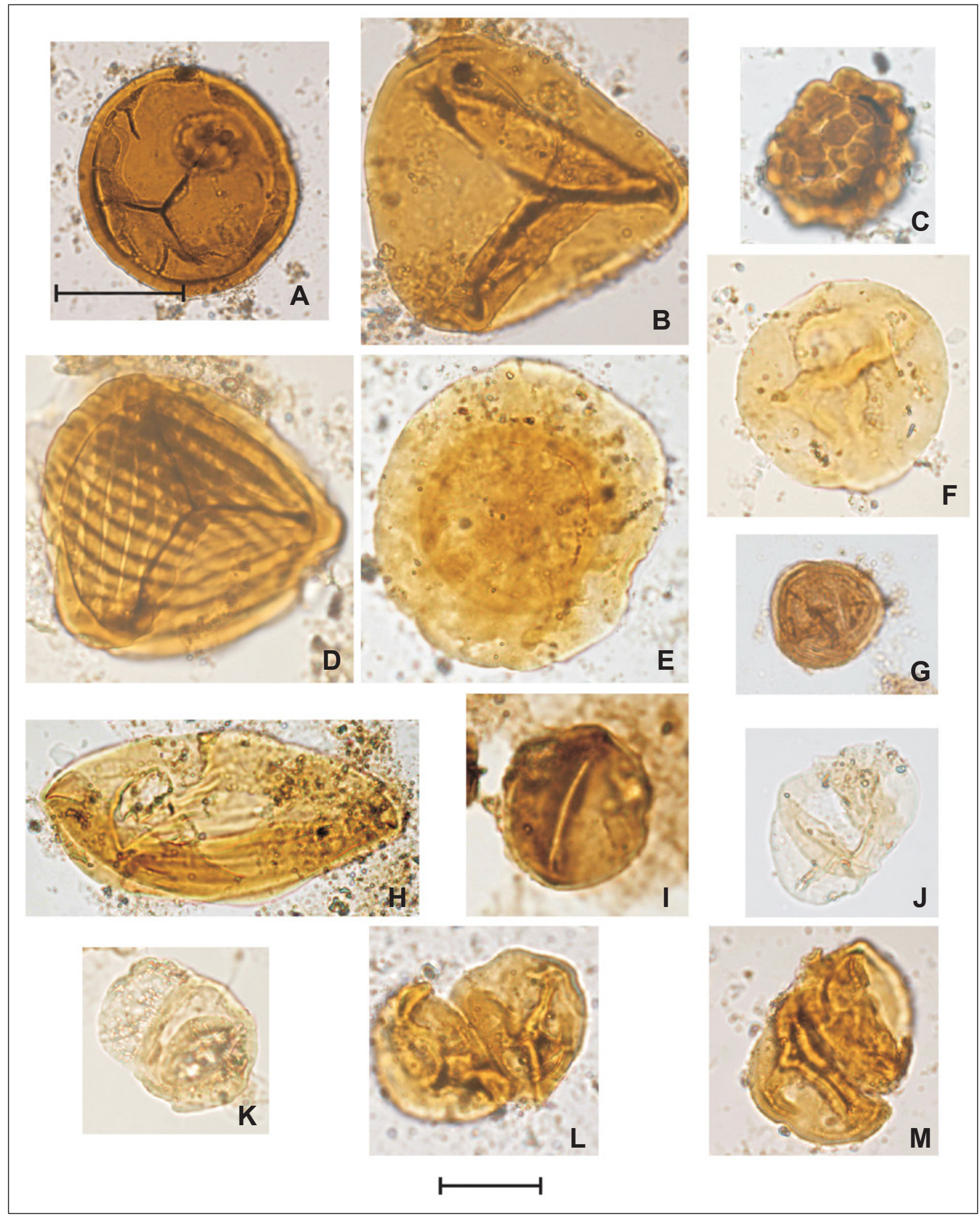

Figura 4/Figure 4. Fotomicrografias representativas de palinomorfos recuperados/Representative of photomicrographs recovered palynomorphs. A, Todisporites cinctus (Orlowskazwolinska) Malyawkina, 1964; B, Deltoidospora hallii Miner, 1935; C, Leptolepidites psarosus Norris, 1968; D, Cicatricosisporites sp.1 Dino, 1992; E, Callialasporites lucidus (Pocock) Reyre, 1973; F, Araucariacites australis Cookson, 1947; G, Classopollis classoides Pflug, 1953; H, Equisetosporites concinnus Singh, 1964; I, Monocolpollenites spheroidites Jardiné \& Magloire, 1965; J,K, Vitreisporites pallidus (Reissinger) Nilsson, 1958; L,M, Dicheiropollis etruscus Trevisan, 1971. Escalas/Scale bars: A = 50 ㅆm; $\mathrm{B}-\mathrm{M}=20 \mu \mathrm{m}$. 


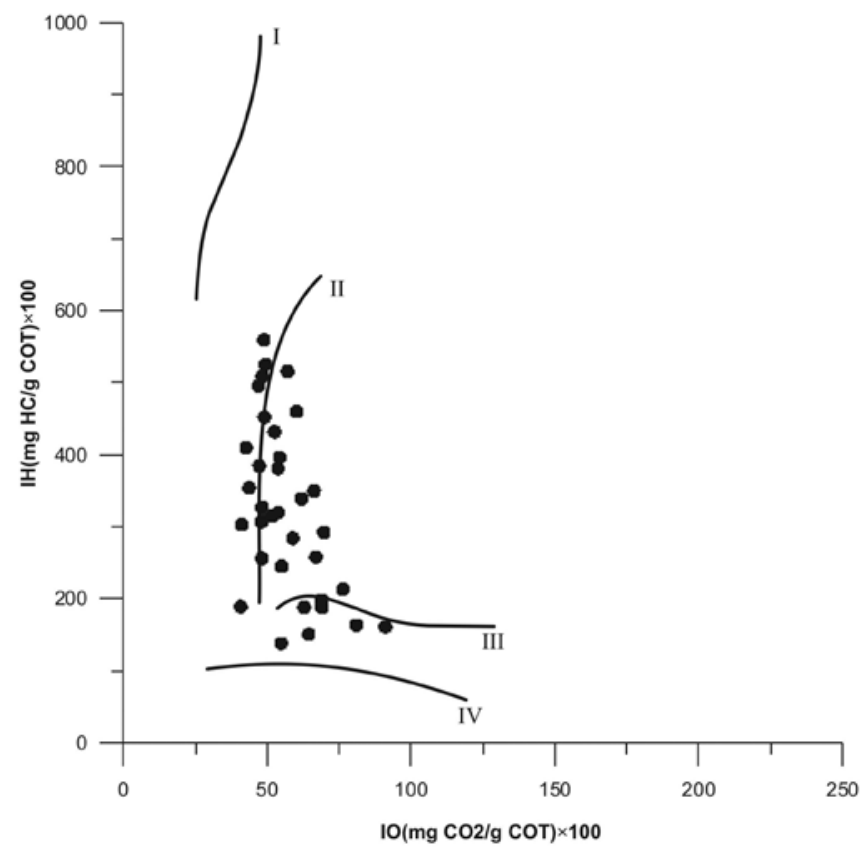

Figura 5. Diagrama binário representando os tipos de querogênio, IH × IO (Espitalié et al., 1977).

Figure 5. Binary diagram representing the types of kerogen, $\mathrm{IH} \times 1 \mathrm{O}$ (Espitalié et al., 1977).

\section{Palinofácies}

Com a integração dos resultados das análises, foi possível individualizar para o presente estudo três palinofácies, denominadas como palinofácies 1, 2 e 3 (Tabela 2).

O grupo da Matéria Orgânica Amorfa (MOA) domina a assembleia dos componentes orgânicos. A MOA, sob luz branca transmitida, apresenta grumos com coloração variando do amarelo ao marrom e sob luz ultravioleta incidente, as colorações de fluorescência variam do verde ao laranja claro (Figura 6).

A elevada abundância relativa de MOA com fluorescência é característica de áreas de alta preservação de matéria orgânica com intenso retrabalhamento microbiológico devido às condições redutoras, especialmente em áreas de alta produtividade primária (Tyson, 1993, 1995). No geral, os demais grupos do querogênio representam baixos a baixíssimos percentuais do total do querogênio. $\mathrm{O}$ grupo dos fitoclastos (derivado de tecidos lenhosos de vegetais superiores) é composto predominantemente de fitoclastos opacos de tamanho diminuto.

A palinofácies 1 é caracterizada por conter excelente contribuição de material orgânico amorfo $(60 \%$ a $90 \%)$, que se apresenta disperso com alto grau de fluorescência, indicando um excelente estado de preservação. A grande abundância de MOA preservada indica um ambiente com condições redutoras, com uma coluna de água anóxica contendo alta produtividade primária, devido a intensa atividade microbiológica. Os raros palinomorfos presentes mostram fluorescência baixa a moderada. Esta palinofácies é encontrada no intervalo $98,80 \mathrm{~m}-76,70 \mathrm{~m}$.
Tabela 2. Resultados de COT (\%) e pirólise Rock-Eval das amostras analisadas do poço 9-FBA-79-BA.

Table 2. Results of the TOC (\%) and "Rock-Eval" pyrolysis of the samples of the 9-FBA-79-BA well.

\begin{tabular}{|c|c|c|c|c|c|}
\hline \multirow{2}{*}{$\begin{array}{c}\text { Amostra } \\
\text { Profundidade } \\
\text { (m) }\end{array}$} & \multirow{2}{*}{$\begin{array}{c}\text { M.O.A. } \\
(\%)\end{array}$} & \multicolumn{2}{|c|}{$\begin{array}{c}\text { Fitoclastos } \\
(\%)\end{array}$} & \multirow{2}{*}{$\begin{array}{c}\text { Palinomorfos } \\
(\%)\end{array}$} & \multirow[b]{2}{*}{ Palinofácies } \\
\hline & & Opacos & Translúcidos & & \\
\hline 31,10 & 50 & 33 & 12 & 5 & III \\
\hline 32,30 & 40 & 28 & 27 & 5 & III \\
\hline 33,50 & 50 & 27 & 18 & 5 & II \\
\hline 43,70 & 70 & 20 & 8 & 2 & II \\
\hline 44,30 & 45 & 34 & 14 & 7 & III \\
\hline 52,10 & 45 & 29 & 16 & 10 & III \\
\hline 53,30 & 45 & 32 & 14 & 9 & III \\
\hline 54,20 & 50 & 23 & 20 & 7 & III \\
\hline 56,30 & 45 & 37 & 11 & 7 & III \\
\hline 59,30 & 45 & 34 & 13 & 8 & III \\
\hline 61,20 & 85 & 9 & 3 & 3 & II \\
\hline 62,10 & 70 & 25 & 4 & 1 & II \\
\hline 63,90 & 90 & 7 & 1 & 2 & II \\
\hline 64,50 & 85 & 9 & 1 & 5 & II \\
\hline 65,00 & 70 & 26 & 3 & 1 & II \\
\hline 66,20 & 90 & 4 & 1 & 5 & II \\
\hline 67,10 & 90 & 4 & 2 & 4 & II \\
\hline 68,00 & 70 & 26 & 3 & 1 & II \\
\hline 73,70 & 70 & 21 & 8 & 1 & II \\
\hline 74,60 & 85 & 11 & 2 & 2 & II \\
\hline 75,50 & 60 & 28 & 6 & 6 & II \\
\hline 76,70 & 80 & 15 & 3 & 2 & I \\
\hline 77,60 & 90 & 7 & 1 & 2 & I \\
\hline 78,50 & 85 & 9 & 1 & 5 & I \\
\hline 79,40 & 85 & 12 & 1 & 2 & I \\
\hline 81,80 & 90 & 3 & 2 & 5 & I \\
\hline 82,70 & 45 & 36 & 14 & 5 & I \\
\hline 85,70 & 85 & 12 & 2 & 1 & I \\
\hline 90,80 & 85 & 12 & 3 & 0 & I \\
\hline 91,70 & 80 & 12 & 2 & 6 & I \\
\hline 92,60 & 80 & 10 & 6 & 4 & I \\
\hline 93,50 & 80 & 17 & 3 & 0 & I \\
\hline 96,50 & 60 & 23 & 15 & 2 & I \\
\hline 98,80 & 60 & 25 & 12 & 3 & I \\
\hline
\end{tabular}




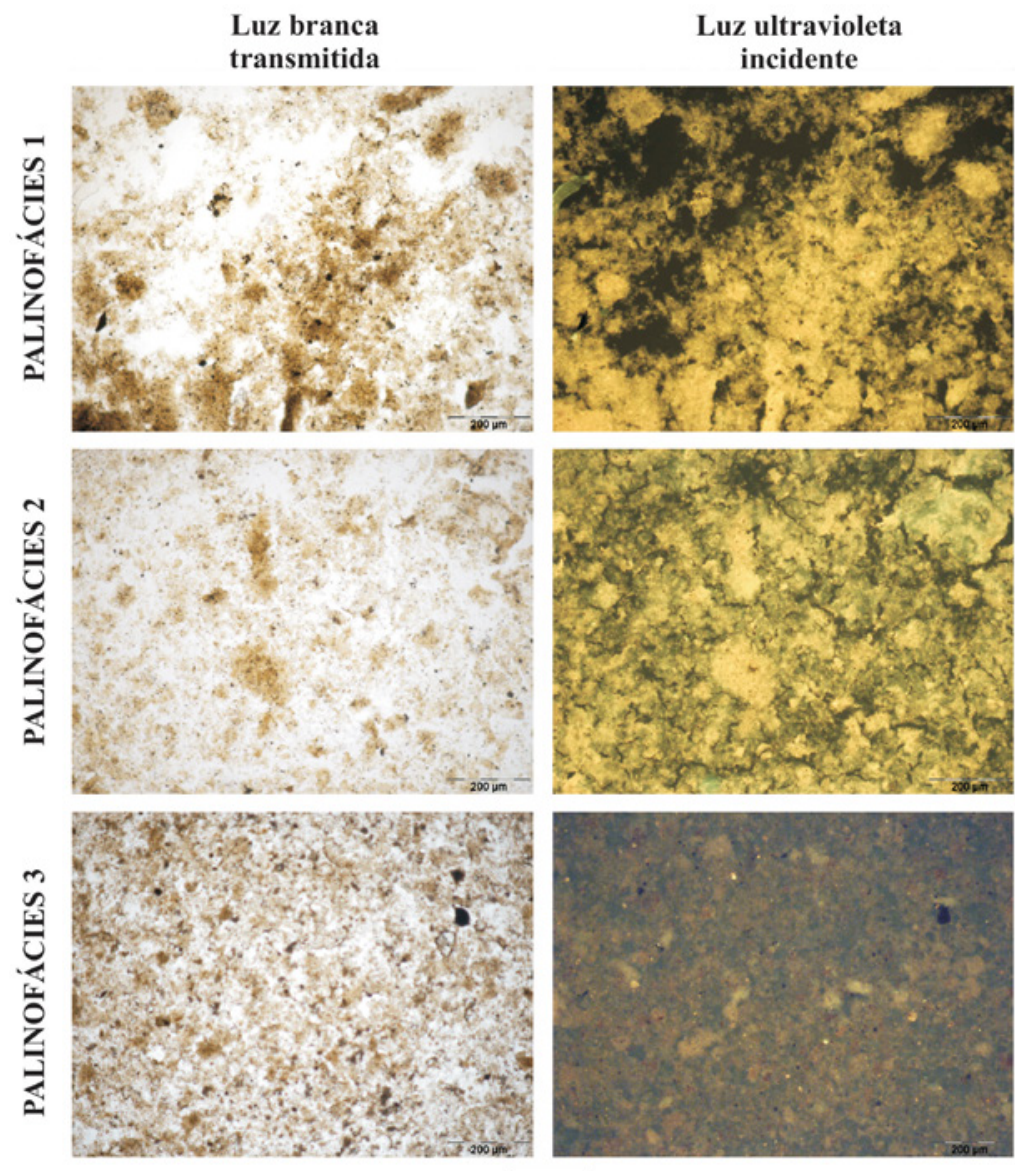

Figura 6. Palinofácies com fotos em luz branca transmitida e em luz ultravioleta incidente. Escalas $=200 \mu \mathrm{m}$.

Figure 6. Palynofacies with photos in transmitted white light and incidental ultraviolet light. Scale bars $=200 \mu \mathrm{m}$.

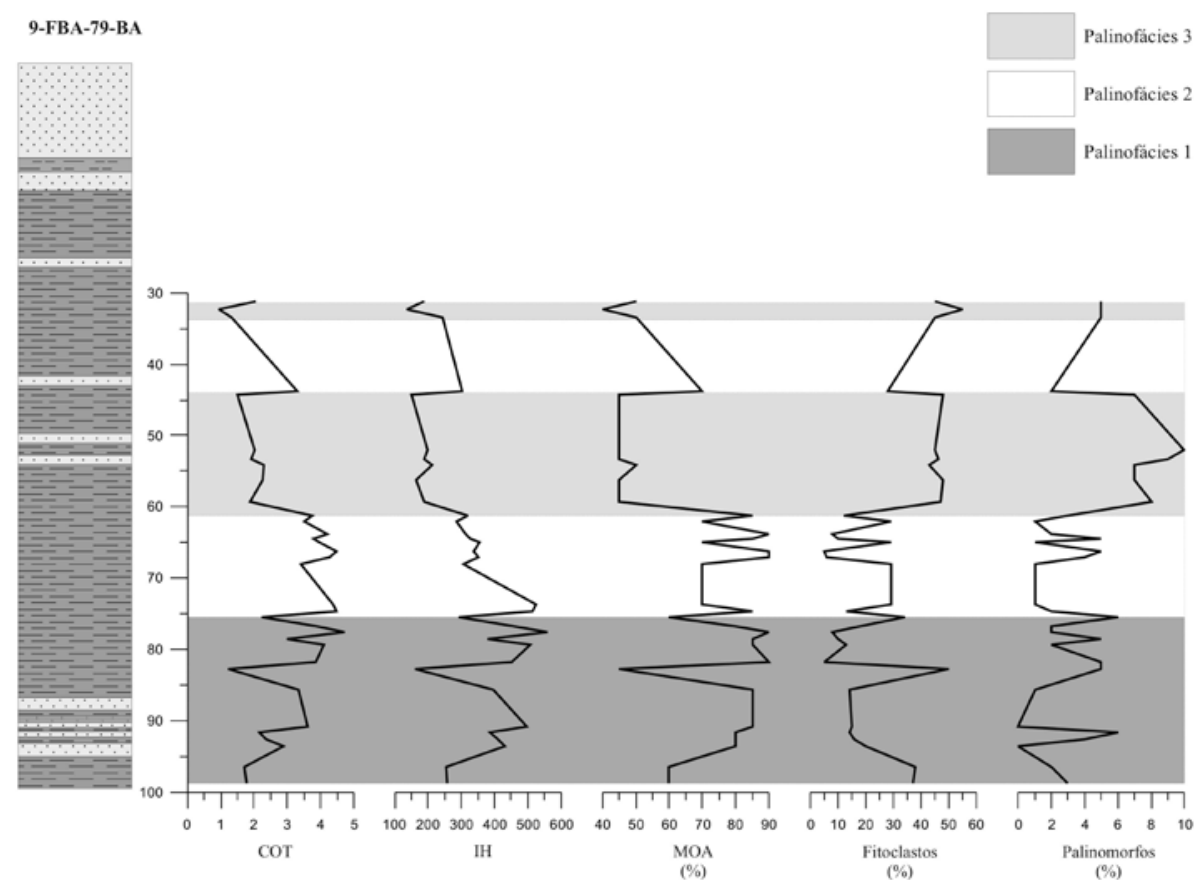

Figura 7. Correlação entre os teores de carbono orgânico total (COT), índice de hidrogênio (IH) e os constituintes do querogênio com os níveis representativos do poço 9-FBA-79-BA.

Figure 7. Correlation between the total organic carbon (TOC), hydrogen index (HI) and kerogen constituents in the 9-FBA-79-BA well. 
A palinofácies 2 possui características semelhantes a palinofácies 1 , porém os fitoclastos proporcionalmente aumentam e a matéria orgânica no geral se encontra dispersa, apresentando fluorescência média a alta em bom estado de preservação e os palinomorfos apresentam baixa fluorescência. Os intervalos correspondentes a esta palinofácies são 75,50 $\mathrm{m}-61,20 \mathrm{~m} ; 43,70 \mathrm{~m} ; 33,50 \mathrm{~m}$.

Em relação à palinofácies anteriores, a palinofácie 3 (nos intervalos 59,30 m-44,30 m; 32,30 m; 31,10 m) apresenta quantidade mais baixa de MOA ( $40 \%$ a $50 \%$ ), aumento da porcentagem dos grupos dos fitoclastos e palinomorfos de origem continental e fluorescência baixa. Isso nos permite inferir que estes componentes de origem continental estariam associados com o transporte fluvial ou eólico que, com a contribuição sedimentar, favoreceria uma possível turbulência da água e do estabelecimento de condições mais oxidantes.

Os palinomorfos, de uma maneira geral, apresentam coloração de amarelo escuro a laranja (principalmente os esporos observados para o ICE), quando observados em luz branca transmitida, mas quando são expostos à luz ultravioleta incidente apresentam fluorescência baixa de coloração amarelada à coloração laranja. Portanto, é possível identificar no material analisado, que a Formação Pojuca se encontra entrando na janela de geração, com valores de ICE 4,5-5,0.

\section{DISCUSSÃO}

As análises de palinofácies, palinologia, COT e pirólise Rock-Eval nos sedimentos pertencentes ao poço 9-FBA79-BA envolveram estudos quantitativos e qualitativos da assembleia dos componentes da matéria orgânica da Formação Pojuca, permitindo estabelecer algumas observações (Figura 7).

No presente estudo são encontrados teores de COT atingindo percentuais de $4,70 \%$, corroborando com teores encontrados em rochas geradoras da Bacia do Recôncavo por Aragão et al. (1998) e Coutinho (2008), que encontraram valores de COT na Formação Candeias variando de $0,8 \%$ a $4 \%$ e $1,5 \%$ a $4,91 \%$. Alguns dos baixos valores nos teores de COT são explicados devido a não preservação da matéria orgânica depositada, ou devido à diluição da matéria orgânica diante de altas taxas de sedimentação.

Com base nos resultados de palinofácies e pirólise RockEval através da utilização do diagrama tipo Van Krevelen, pôde-se classificar a matéria orgânica como sendo dos tipos II e III para o intervalo estudado, sendo a sua grande maioria constituída pelo querogênio do tipo II, rica em hidrogênio e pobre em oxigênio. Mello et al. (1994), trabalhando em rochas geradoras de origem lacustre nesta mesma bacia, caracterizaram a matéria orgânica dessas rochas geradoras como querogênio do tipo I, já Daniel et al. (1989) e Balbinot \& Kalkreuth (2010) identificaram como querogênio do tipo II e III, sugerindo uma mistura de matéria orgânica com contribuição terrestre e lacustre.

Talbot (1988), por exemplo, observou um predomínio de querogênio do tipo II nos sedimentos recentes de alguns lagos do sistema rifte do leste Africano e Ariztegui et al. (1994) também reportaram uma alternância de querogênios dos tipos I e II nos sedimentos de lagos na Suíça, atribuindo o fato a variações nas condições físico-químicas da coluna d'água e na natureza da biota dominante (diatomáceas vs. dinoflagelados). A presença em variadas proporções de querogênio do tipo III também tem sido reportada por diversos autores em diferentes sistemas lacustres (e.g. Powell, 1986; Horsfield et al., 1994). Isso acontece porque em ambientes sedimentares próximos de fontes flúvio-deltaicas, observa-se uma alta taxa de acumulação de sedimentos, incorporando uma grande quantidade de matéria orgânica alóctone (derivada de plantas terrestres), fator que implica na diluição da matéria orgânica autóctone (derivada de algas) (Bustin, 1988).

No presente trabalho foram identificadas palinofácies com boas condições de preservação da matéria orgânica. A análise visual indicou que as amostras estudadas possuem uma mistura de MO lacustre e continental. Contudo, observou-se que a MOA representa o componente orgânico mais abundante por amostra, indicando que as palinofácies encontram-se inseridas em unidades sedimentares lacustres (Portela, 2012), onde é muito frequente a MOA representar o componente mais abundante, devido à grande disponibilidade de $\mathrm{MO}$ autóctone que é fornecida pelo fitoplâncton (Tyson, 1995).

Os esporomorfos estão representados na sua grande maioria por grãos de pólen de Classopollis classoides, grãos de pólen bissacados e esporos, o que demonstra uma associação que caracteriza origens distintas. Os grãos de Classopollis provêm de uma vegetação de coníferas de pequeno porte da família Cheirolepidiaceae, extinta no Thanetiano (Paleoceno superior) (Quattrocchio, 2006), com significado ecológico e paleoclimático muito característico, pois possuíam capacidade de adaptação a condições de salinidade e clima quente e seco (Vakhrameev, 1991; Lima, 1978; Batten, 1974). Sendo a população mais abundante de esporomorfos e presente em quase todas as amostras, esta família de coníferas provavelmente ocupava uma posição muito próxima à área de sedimentação.

Os grãos de pólen bissacados possuem dois sacos aéreos e podem deslocar-se da fonte ao ambiente deposicional por longos períodos de tempo por ação do vento e por flutuação na água (Hopkins, 1950). A ocorrência destes grãos de pólen sugere que provêm de uma vegetação relativamente afastada, ou seja, desenvolvida numa posição mais distal e mais favorável ao crescimento de vegetação com árvores de grande porte. Se encontram particularmente associados aos horizontes mais ricos em fitoclastos, o que reforça uma proveniência a partir de uma fonte mais continental.

A associação lacustre representada pelas algas Pediastrum e Botryococcus não são encontradas nas amostras estudadas, devido ao alto grau de amorfização corroborado com os altos índices de MOA nas amostras estudadas.

Os valores do ICE estabelecidos para o poço 9-FBA79-BA apresentaram variações, ao longo de toda a seção, em torno de 4,5-5,0 de ICE, que corresponde a $0,5 \%$ da Reflectância da Vitrinita (\%Ro). Utilizando-se a tabela padrão da Robertson Research como referência de maturação, foi possível caracterizar o material orgânico entrando na janela de geração de hidrocarbonetos para os poços estudados. 


\section{CONCLUSÕES}

Através dos resultados das análises dos principais constituintes orgânicos, foi possível reconhecer três distintas palinofácies: Palinofácies 1 (P1), Palinofácies 2 (P2) e Palinofácies 3 (P3); dentre as palinofácies individualizadas, destaca-se a P1 e P2 por apresentar maior proporção de MOA, com alto grau de fluorescência, valores de COT atingindo percentuais de 4,70\% e valores de 4,5-5,0 de ICE, indicando um bom potencial para geração de hidrocarbonetos.

Mediante aos resultados apresentados foi possível identificar uma ciclicidade comum na Formação Pojuca, na qual se alternam depósitos de sistemas lacustres transgressivos e regressivos devido a ocorrência de flutuações periódicas da lâmina d'água, resultando em três diferentes ambientes deposicionais para a época desta seção: lago central, lago transicional e lago marginal. Os teores de COT e a quantidade de matéria orgânica observada estão relacionados com a oxigenação da coluna d'água, uma vez que os valores elevados de carbono orgânico total também estão relacionados aos maiores valores de $\mathrm{IH}$, indicando condições de anoxia do ambiente de sedimentação lacustre.

Com a integração dos dados adquiridos por meio das análises geoquímicas, das palinofácies e associações palinológicas, concluiu-se que a sedimentação da Formação Pojuca é caracterizada por um ambiente de deposição continental (deltaico-lacustre). A presença de elementos higrofíticos e xerofíticos indica uma vegetação que se desenvolveu num paleoclima árido, com nichos úmidos localmente e de latitude próxima ao atual. Analisando o material orgânico preservado, foi possível concluir que o mesmo foi depositado em condições de anoxia, contendo querogênio de boa qualidade e adequado para a geração de hidrocarbonetos.

\section{AGRADECIMENTOS}

Os autores agradecem a Petrobras pelas amostras cedidas, a Agência Nacional do Petróleo (ANP) pelo apoio financeiro, e aos laboratórios de Palinomacerais (LBPM) e Geoquímica (LGQM) da Universidade do Estado do Rio de Janeiro pelas análises das amostras utilizadas neste estudo.

\section{REFERÊNCIAS}

Aragão, M.A.N.F; Trindade, L.A.F.; Araújo, C.V.; Silva, O.B.; Scartezini, A.A.; Oswald, F.H.; Canario, J.A. \& Garcia A.P. 1998. Distribution and controls of lacustrine source rocks in the Recôncavo Basin, Brazil. American Association of Petroleum Geologists Bulletin, 82:1886.

Ariztegui, D.; Hollander, D.J. \& Mckenzie, J.A. 1994. Algal dominated lacustrine matter can be either type I or type I: evidence for biological, chemical and physical controls on organic matter quality. In: LATIN AMERICAN CONGRESS ON ORGANIC GEOCHEMISTRY, 4, 1994. Resumos Expandidos, Bucaramanga, p.25-27.

ASTM. 2008. American Society for Testing and Materials. Standard test methods for Sulfur in the analysis sample of coal and coke using high-temperature tube furnace combustion methods ASTMD 4239. Disponível em http://engineers.ihs.com/ documents/abstract/NWETIBA; acessado em 03/08/2016.

Balbinot, M. \& Kalkreuth, W. 2010. Organic geochemistry and petrology of the Gomo Member, Recôncavo basin, Brazil. International Journal of Coal Geology, 84:286-292. doi:10.1016/j.coal.2010.09.008

Barnard, P.C.; Collins, A.G. \& Cooper, B.S. 1981. Identification of Kerogen Facies in a Source Rock Horizon. Examples from the North Sea Basin. In: J. Brooks (ed.) Organic Maturation Studies and Fossil Fuel Exploration, Academic Press, p. 271-282.

Batten, D.J. 1974. Wealden palaeoecology from the distribution of plant fossils. Proceedings of the Geologists' Association, 85:433-458.

Behar, F.; Beaumont, H.L. \& Penteado, H.L. 2001. Rock-Eval 6 Technology: Performance and Developments. Oil \& Gas Science Technology - Revue d'IFP, 56:111-134. doi:10.2516/ ogst:2001013

Beurlen, G. et al. 1992. Bioestratigrafia das bacias mesozóicascenozóicas brasileiras: texto explicativo das cartas bioestratigráficas. Rio de Janeiro, PETROBRAS/CENPES/ DIVEX/SEBIPE, 616 p. (Relatório interno).

Bustin, R.M. 1988. Sedimentology and characteristics of dispersed organic matter in Tertiary Niger Delta: origin of source rocks in a deltaic environment. American Association of Petroleum Geologists Bulletin, 72:277-298.

Caixeta, J.M.; Bueno, G.V.; Magnavita, L.P. \& Feijó, F.J. 1994. Bacias do Recôncavo, Tucano e Jatobá. Boletim de Geociências da Petrobrás, 8:163-172.

Coutinho, L.F.C. 2008. Análise do Balanço Material do Petróleo em uma Região em Fase de Exploração Matura - Bacia do Recôncavo, Brasil. Universidade Federal do Rio de Janeiro, Tese de doutorado, $431 \mathrm{p}$.

Daniel, L.M.F.; Souza, E.M. \& Mato, L.F. 1989. Geoquímica e modelos de migração de hidrocarbonetos no Campo de Rio do Bú: integração com o Compartimento Nordeste da Bacia do Recôncavo, Bahia. Boletim de Geociências da Petrobrás, 3:201-214.

Destro, N.; Szatmari, P.; Alkmim, F.F. \& Magnavita, L.P. 2003. Release faults, associated structures, and their control on petroleum trends in the Recôncavo rift, northeast Brazil. American Association of Petroleum Geologists Bulletin, 87:1123-1144.

Espitalié, J.; Laporte, J.L.; Madec, M.; Marquis, F.; Lepat, P.; Paulet, J. \& Boutefeu, A. 1977. Méthode rapide de caractérisation des roches mère, de leur potential pétrolier et de leur degree d'évolution. Revue de l'Institute Français du Pétrole, 32:23-42.

Figueiredo, A.M.F.; Braga, J.A.E.; Zabalaga, J.C.; Oliveira, J.J.; Aguiar, G.A.; Silva, O.B.; Mato, L.F.; Daniel, L.M.F.; Magnavita, L.P. \& Bruhn, C.H.L. 1994. Recôncavo Basin, Brazil: a prolific intracontinental Rift Basin. In: S.M. Landon (ed.) Interior Rift Basins, Tulsa, American Association of Petroleum Geologists, p. 157-203. (Memoir 59).

Gaglianone, P.C. \& Trindade, L.A.F. 1988. Caracterização geoquímica dos óleos da Bacia do Recôncavo. Geochimica Brasiliensis, 2:15-39.

Hernegreen, G.F.W.; Kedves, M.; Rovnina, L.V. \& Smirnova, S.B. 1996. Cretaceous palynofloral provinces: a review. In: J. Jansonius \& D.C. McGregor (eds.) Palynology, Principles and Applications, v. 3, American Association of Stratigraphic Palynologists Foundation, p. 1157-1188.

Hopkins, J.S. 1950. Differential flotation and deposition of coniferous and deciduous tree pollen. Ecology, 31:633-641. 
Horsfield, B. et al. 1994. Organic geochemistry of freshwater and alkaline lacustrine sediments in the Green River Formation of the Washakie Basin, Wyoming, USA. Organic Geochemistry, 22:415-440. doi:10.1016/0146-6380(94)90117-1

Lima, M.R. 1978. Palinologia da Formação Santana (Cretáceo do Nordeste do Brasil). Instituto de Geociências, Universidade de São Paulo, Tese de doutorado, 338 p.

Magnavita, L.P.1992. Geometry and kinematics of the RecôncavoTucano-Jatobá Rift, Northeast Brazil. University of Oxford, Tese de doutorado, $493 \mathrm{p}$.

Magnavita, L.P.; Silva, R.R. \& Sanches, C.P. 2005. Guia de campo da Bacia do Recôncavo, NE do Brasil. Boletim de Geociências da Petrobras, 13:301-334.

Mello, M.R. \& Maxwell, J.R. 1990. Organic Geochemical and biological marker characterization of source rocks and oils derived from lacustrine environments in the Brazilian continental margin. In: B.J. Katz (ed.) Lacustrine Basin Exploration: Case Studies and Modern Analogs, Tulsa, American Association of Petroleum Geologists, p. 7-98 (Memoir 50).

Mello, M.R.; Mohriak, W.U.; Koutsoukos, E.A.M. \& Bacoccoli, G. 1994. Selected petroleum systems in Brazil. In: L.B. Magoon \& W.G. Dow (eds.) The Petroleum System - from Source to Trap, Tulsa, American Association of Petroleum Geologists, p. 499512 (Memoir 60).

Mendonça Filho, J.G.; Carvalho, M.A. \& Menezes, T.R. 2002. Palinofácies. In: T.L. Dutra (ed.) Técnicas e Procedimentos para o Trabalho com Fósseis e Formas Modernas Comparativas, Unisinos, p. 20-24.

Portela, H.A. 2012. Caracterização da matéria orgânica potencialmente geradora de petróleo e seu posicionamento Bioestratigráfico com base em Palinologia e Palinofácies na região de Aramari - Bacia do Recôncavo. Programa de Análise de Bacias, Universidade do Estado do Rio de Janeiro, Tese de doutorado, $156 \mathrm{p}$.

Powell, T.G. 1986. Petroleum geochemistry and depositional setting of lacustrine source rocks. Marine and Petroleum Geology, 3:200-219. doi:10.1016/0264-8172(86)90045-0

Quattrocchio, M. 2006. Palynology and Palaeocommunities of the Paleogene of Argentina. Revista Brasileira de Paleontologia, 9:101-108.

Schumacher, B.A. 2002. Methods for the Determination of Total Organic Carbon (TOC) in Soils and Sediments. Las Vegas, Ecological Risk Assessment Support Center, Office of Research and Development, 23 p. (Report NCEA-C-1282).

Silva, O.B. da; Caixeta, J.M.; Milhomem, P.S \& Kosin, M.D. 2007. Bacia do Recôncavo, Boletim de Geociências da Petrobras, 15:423-431.

Talbot, M.R. 1988. The origin of lacustrine source rocks: evidence from the lakes of tropical Africa. In: A.J. Fleet; K. Kelts \& M.R. Talbot (eds.) Lacustrine petroleum source rocks, Oxford, Geological Society, p. 29-43 (Special Publication 40).

Traverse, A. 1988. Paleopalynology. London, Unwin Hyman, 600 p. Tyson, R.V. 1993. Palynofacies Analysis. In: D.G Jenkins (ed.) Applied Micropaleontology, Kluwer Academic Publishers, p. 153-191.

Tyson, R.V. 1995. Sedimentary organic matter: organic facies and palynofacies. $1^{\mathrm{a}}$ ed. London, Chapman \& Hall, $615 \mathrm{p}$.

Vakhrameev, V.A. 1991. Jurassic and Cretaceous floras and climates of the Earth. Cambridge, Cambridge University Press, 318 p.

Received in January, 2016; accepted in June, 2016. 